\title{
Evaluation of the pre-bariatric surgery weight management service during COVID-19 lockdown in March 2020, UK
}

\author{
A.N. Suleiman ${ }^{1}$, S.S. Rasoul ${ }^{1}$ and D. Bhakta ${ }^{2}$ \\ ${ }^{1}$ Nutrition and Dietetics Bariatric Service, Chelsea and Westminster NHS Foundation Trust, London, UK and \\ ${ }^{2}$ Public Health Nutrition Research Group, London Metropolitan University, London, UK
}

Obesity prevalence is a growing public health issue in England due to its association with chronic diseases ${ }^{(1)}$. Exacerbated by the pandemic, obese groups were identified as high-risk to COVID-19 ${ }^{(2)}$. High transmission rate of COVID-19 enforced restrictions and lockdown measures $^{(3)}$ in March 2020. NHS services adapted to limit transmission, subsequently cancelling face-to-face appointments. This potentially created additional barriers to promote successful weight management in individuals being counselled for weight loss before bariatric surgery. The service at Chelsea and Westminster Hospital consists of nutritional and lifestyle support from a multidisciplinary team. Participants attend two compulsory group sessions covering areas in healthy eating based on portion control, mindful eating, healthy snacks and meal alternatives, reading and using food labels and techniques to overcome barriers to a healthier lifestyle. This is followed by one-to-one consultations to assess current weight loss or maintenance, adherence to recommendations covered in group sessions and creating a personalised plan.

This project is based on a qualitative and quantitative retrospective service evaluation study design. The NHS CERNER system was used to screen patient records to assess eligibility and allow data collection of body weights, body mass index, dietary intake and demographics. Data was collected from three periods during the pandemic before the first COVID-19 lockdown (January March 2020), during (April - May) and after (June - July 2020). A calibrated bariatric weighing scale (Seca645 Class III) was used to measure, body weight; this was collected before lockdown. Dietary assessments (24hour recalls and FFQ, measuring specific food items e.g. crisps / pastries) and reported body weight collected between April - July 2020 were from virtual assessments either by telephone or video consultations. A questionnaire was also used to collect information on participants' access to food, shopping and eating behaviours during lockdown. Dietary assessment was analysed using Nutritics. Statistical significance was set at $\mathrm{p} \leq 0.05$.

A total of 51 participants were included in the study with a mean age (years) of $45.5 \pm 10.3$ and a mean BMI (kg/m2) $43.8 \pm 5.8$. No statistical differences were observed in energy, macro-and micronutrient intake before, during and after lockdown, however significant decreases in BMI were observed during and after lockdown, mean BMI, $-1.85 \pm 2.5(\mathrm{p}=0.03)$. Difficulties in following dietary advice during lockdown were associated with difficulty in accessing healthier foods $(p=0.004)$ and ready meals $(p=0.04)$.

Individuals receiving weight management support during and after lockdown had positive outcomes in weight loss despite reported difficulties in following dietary recommendations during lockdown. Additionally, inability of going to the supermarket and increased reliance on others for access to food may have positively influenced BMI reduction, possibly due to limited control on food choices and quantity.

\section{References}

1. Agha M \& Agha R (2017) Int J Surg Onol 2(7), 17

2. Banerjee A, Pasea L, Harris S, et al. (2020) Lancet 395, 1715-1725.

3. Rajendran DK, Rajagopal V, Alagumanian S, et al. (2020) Virus disease 31, 161-173. 